\title{
A acessibilidade arquitetônica interfere na usabilidade de indivíduos com mobilidade reduzida?
}

\section{Does accessibility interfere in usability of people with restricted mobility?}

\author{
Bárbara Guerra Salles ${ }^{1}$, Flora Pereira Guerra ${ }^{1}$, Érika Akemi Soki ${ }^{2}$, \\ Mariana Luiza Guimarães Costa ${ }^{2}$, Márcia Bastos Rezende ${ }^{3}$
}

SALLES, B. G.; GUERRA, F. P.; SOKI, E. A.; COSTA, M. L. G.; REZENDE, M. B. A acessibilidade arquitetônica interfere na usabilidade de indivíduos com mobilidade reduzida? Rev. Ter. Ocup. Univ. São Paulo, v. 21, n. 1, p. 83-88, jan./abr. 2010.

RESUMO: Um dos focos da intervenção terapêutica ocupacional refere-se à inclusão de indivíduos em atividades de sua vida cotidiana, com o máximo de independência e autonomia possíveis. Além da avaliação do espaço físico (acessibilidade), cabe ao profissional verificar a usabilidade, que consiste na avaliação subjetiva do indivíduo sobre como o ambiente restringe suas atividades. Realizou-se este estudo com o intuito de investigar, sob o ponto de vista dos usuários com mobilidade reduzida (MR), se as condições arquitetônicas interferem nas atividades e participação dos mesmos na Escola de Educação Física, Fisioterapia e Terapia Ocupacional (EEFFTO) da Universidade Federal de Minas Gerais (UFMG), além de averiguar a satisfação desses em relação ao suporte oferecido pela UFMG e suas sugestões de adequação. Para tanto, desenvolveu-se um estudo descritivo, do tipo survey. Aplicou-se um questionário, no período de maio a setembro de 2008, para uma amostra de 26 indivíduos. O questionário contém 4 questões abertas e 21 questões fechadas. As questões iniciais eram para descrever a amostra, enquanto as demais abordaram o acesso e o uso do espaço físico. Quanto à acessibilidade da EEFFTO em geral, $73 \%$ dos indivíduos classificaram como tendo "Ótimas condições de acesso". Isso refletiu a satisfação da maioria dos usuários neste aspecto, apesar do não atendimento integral às regras da ABNT já ter sido constatado em estudo preliminar; logo, os resultados sugerem que as barreiras arquitetônicas não interferem na usabilidade desses usuários.

DESCRITORES: Limitação da mobilidade. Estruturas de acesso/normas. Avaliação da deficiência. Defesa das pessoas com deficiência. Acesso aos serviços de saúde.

\footnotetext{
* Parte integrante do Trabalho de Conclusão de Curso das estudantes Bárbara Salles e Flora Guerra. Este trabalho foi apresentado nos seguintes eventos:

-XIII ENAPET (Encontro Nacional dos grupos PET), Campinas: 14 a 18 de julho de 2008.

-XVII Semana da Iniciação Científica, Universidade Federal de Minas Gerais: 20 a 24 de outubro de 2008.

-VII Congresso Norte Nordeste de Terapia Ocupacional, Salvador: 25 a 28 de novembro de 2008.

1. Terapeutas ocupacionais graduadas pela UFMG e egressas do PET Terapia Ocupacional - UFMG

2. Fisioterapeutas graduadas pela UFMG

3. Prof $^{\mathrm{a}} \mathrm{Dr}^{\mathrm{a}}$ do Departamento de Terapia Ocupacional da UFMG e tutora do PET Terapia Ocupacional - UFMG.

Endereço para correspondência:Dra. Márcia Bastos Rezende. Rua Orozimbo Nonato, 901/3. Bairro Dona Clara - Belo Horizonte-Minas Gerais - CEP 31260-230
} 


\section{INTRODUÇÃO}

$\mathrm{N}$

os últimos anos, oportunidades iguais para todas as pessoas estão sendo enfatizadas

e a acessibilidade e a usabilidade vêm recebendo atenção crescente da sociedade. A importância de se ter uma vida independente está sendo cada vez mais reconhecida (IWARSSON; STAHL, 2003; FANGE e IWARSSON, 2003), e um dos objetivos da Terapia Ocupacional é justamente investir para melhorar a inclusão dos indivíduos a todos os aspectos que englobam sua vida cotidiana, garantindo maior independência e autonomia no desempenho de seus papéis (RIBEIRO, 2007). Assim, torna-se importante estudar a acessibilidade e usabilidade e a relação entre elas, para contribuir na inclusão de todos os indivíduos nos diversos ambientes.

Tendo em vista a relevância do papel da Universidade na construção da cidadania, deve-se discutir como essa garante acesso, permanência e participação de usuários com mobilidade reduzida (MR), independente do papel desempenhado na mesma, sejam docentes, discentes, funcionários ou usuários dos projetos de extensão. Indivíduos com mobilidade reduzida são caracterizados como aqueles que, "temporária ou permanentemente, têm limitada sua capacidade de relacionar-se com o meio e de utilizá-lo. Entende-se por pessoa com mobilidade reduzida, a pessoa com deficiência, idosa, obesa, gestante entre outros" (ABNT / NBR 9050: 2004).

Acessibilidade, segundo Fange e Iwarsson (2003), é um conceito relativo, é o encontro entre a capacidade funcional (componente do indivíduo) e as demandas do ambiente físico (componente ambiental). Ela pode ser dividida em diferentes níveis, são eles: (1) nível micro - refere-se ao ambiente imediato, como a casa e suas proximidades; (2) nível médio - refere-se à vizinhança, como ambientes externos públicos e facilidades públicas na comunidade local ou cidade (por exemplo, transporte público); e (3) nível macro - compreende a sociedade como um todo, toda a nação ou mundo (IWARSSON; STAHL, 2003). No Brasil, a Associação Brasileira de Normas Técnicas (ABNT) define as normas de acessibilidade a construções, mobiliário, espaços e equipamentos urbanos (BRITO et al., 2006). Segundo a mesma, promover acessibilidade significa remover barreiras arquitetônicas, urbanísticas ou ambientais que impeçam a aproximação, transferência ou circulação (ABNT / NBR 9050:2004). Já a usabilidade, palavra freqüentemente utilizada em paralelo com "acessibilidade", é um tema com poucas publicações e significa que a pessoa deveria ser capaz de usar o ambiente de forma igualitária aos demais cidadãos. As condições arquitetônicas devem permitir que qualquer indivíduo, independentemente de qualquer incapacidade, seja capaz de realizar suas atividades diárias. Em outras palavras, "usabilidade" remete a realização dos requisitos funcionais (IWARSSON; STAHL, 2003). A usabilidade é orientada mais subjetivamente que a acessibilidade (FANGE e IWARSSON, 2003). O indivíduo interpreta e avalia o grau com o qual o ambiente restringe e suporta a satisfação de seus objetivos e desejos, normalmente sem reflexões sobre as normas oficiais (IWARSSON; STAHL, 2003). Ela inclui ainda um terceiro componente: a atividade, que consiste no repertório pessoal de atividades do indivíduo, relacionado a situações específicas (FANGE; IWARSSON, 2003).

Realizou-se uma pesquisa anterior com o objetivo de verificar se a Escola de Educação Física, Fisioterapia e Terapia Ocupacional (EEFFTO) da Universidade Federal de Minas Gerais (UFMG) está preparada para receber, viabilizar estudos de permanência e realização de atividades por usuários com MR, considerando a acessibilidade arquitetônica. Essa Escola foi escolhida como alvo da pesquisa por abrigar três cursos na área da saúde, além de diversos projetos de pesquisa e extensão que têm como público alvo os indivíduos com MR. Para tal fim, as condições de acessibilidade da EEFFTO foram analisadas de acordo com as recomendações descritas na norma NBR 9050 da ABNT. $\mathrm{O}$ levantamento arquitetônico de suas dependências e vias de acesso reflete que o avanço da legislação vigente não é acompanhado por mudanças a favor da acessibilidade de pessoas com MR. Detecta-se um não-atendimento a diversas recomendações da NBR 9050 na construção dos prédios da Escola e de seu mobiliário. E evidencia-se ainda uma diferença entre a parte recém construída e a parte antiga da EEFFTO. Por exemplo: todos os banheiros acessíveis segundo as normas estão localizados na parte recém-construída. $\mathrm{O}$ trajeto do estacionamento até a entrada do edifício da parte antiga apresenta um piso bastante irregular, rampa com inclinação inadequada e ausência de corrimão, já o da parte recém-construída apresenta duas rotas de acesso, uma atende a todas as especificações e a outra necessita somente de corrimão em parte de sua extensão (SOKI e COSTA, 2007). Devido a este resultado, decidiu-se ampliar a pesquisa para o âmbito da usabilidade, com o intuito de verificar, sob o ponto de vista dos usuários com $\mathrm{MR}$, se as condições arquitetônicas interferem nas atividades e participação dos mesmos na Escola, além de conhecer a satisfação em relação ao suporte oferecido pela UFMG e suas sugestões de adequação.

\section{METODOLOGIA}

Este é um estudo descritivo, do tipo survey. O projeto foi encaminhado e aprovado pelo Comitê de Ética em Pesquisa da UFMG (Parecer n. ETIC 331/07) e a coleta de 
dados foi autorizada pelo coordenador do Centro de Estudos do Esporte para Portadores de Deficiência (CEPODE), localizado na EEFFTO. Os participantes receberam e assinaram o termo de consentimento livre e esclarecido, que elucida os objetivos e os procedimentos da pesquisa. O questionário, que contém quatro questões abertas e 21 questões fechadas, foi aplicado no período de maio a setembro de 2008. Ele aborda o acesso e a qualidade do mesmo segundo a opinião dos usuários, além das questões iniciais que têm o intuito de descrever a amostra. A primeira parte do questionário conta com perguntas referentes à acessibilidade dos usuários às dependências da Escola, como banheiros, bebedouros, cantina; enquanto a segunda parte é composta por questões que dizem respeito ao uso dessas dependências. As questões fechadas foram elaboradas com base na escala Likert, assim haviam 5 opções de resposta para investigar qual a opinião dos participantes sobre o quão acessível são as instalações do prédio e o quanto de ajuda necessitavam na utilização destas. As opções de respostas eram as seguintes: (1) "ótimas condições de acesso"/"ótimo, administro tudo sozinho"; (2) "boas condições, mas necessita de melhoras"/"bom, administro quase tudo sozinho"; (3) "condições de acesso ruins, oferece muita dificuldade"/"regular, administro algumas coisas sozinho"; (4) "inacessível"/"ruim, preciso de ajuda na maioria das coisas" e (5) "nunca utilizo"/"outro". As perguntas englobam os três andares das partes antiga e nova da Escola.

Foi utilizada uma amostra de conveniência com 26 indivíduos que apresentam mobilidade reduzida, freqüentadores do CEPODE/UFMG (localizado no segundo andar da parte antiga do prédio). Os critérios de inclusão da amostra foram: homens e mulheres acima de 18 anos de idade, sem restrições à raça e classe social; participação nas atividades do CEPODE; e que atendam aos critérios de classificação para mobilidade reduzida segundo a NBR 9050 da ABNT. E como critério de exclusão, os entrevistados não poderiam possuir déficit cognitivo, nem problemas de linguagem e fala. Dentre os entrevistados, $13(50 \%)$ utilizam algum dispositivo de assistência de mobilidade, como bengala, muleta e andador.

Para analisar os resultados, tirou-se a porcentagem de respostas dadas em relação ao total da amostra nas questões fechadas.

\section{RESULTADOS E DISCUSSÃO}

No que se refere ao perfil da amostra utilizada nesse estudo, constatou-se que dos 26 indivíduos entrevistados, oito $(30,77 \%)$ são do sexo feminino e $18(69,23 \%)$ são do sexo masculino. A média etária dos entrevistados é de 50 anos e a maioria dos indivíduos $(65,38 \%)$ têm mobilidade reduzida devido a seqüelas de acidente vascular encefálico, apresentando função reduzida dos membros e por vezes necessitando de dispositivo de assistência para mobilidade.

Os resultados referentes à percepção de acessibilidade do usuário na EEFFTO merecem destaque quanto às classificações do segundo andar do prédio, em que se encontra o CEPODE. As questões relativas a esse andar foram respondidas por todos os usuários do projeto, enquanto as questões relativas aos demais andares do prédio não foram contempladas com outra classificação senão "outro" ou "nunca utilizo"- em sua maioria, dado o desconhecimento ou o pouco uso desses andares por parte dos usuários (Tabela 2 e 3). As razões do não uso ou desconhecimento do segundo e terceiro andares não foram investigadas nesse estudo. Desse modo, as avaliações da percepção da acessibilidade e da usabilidade do primeiro e do terceiro andares encontram-se comprometidas e não serão consideradas neste estudo.

No que se refere às questões relativas ao segundo andar (Tabela 1), o trajeto do estacionamento até a entrada deste andar foi considerado pela maioria dos usuários como tendo "boas condições de acesso, mas necessita de melhoras" (61,5\%). A necessidade de melhorias por eles explicitada vai ao encontro das irregularidades apresentadas no levantamento arquitetônico da Escola, em que, por exemplo, nem todas as rotas de acesso atendem aos requisitos dispostos pela $\mathrm{ABNT}$, como a inclinação de rampas, ou a presença de corrimãos em todas elas (SOKI; COSTA, 2006). Apesar da necessidade de melhoras destacada pela maioria dos usuários, eles ainda classificam esse trajeto como tendo boas condições de acesso.

O trajeto da entrada do segundo andar até o CEPODE e o trajeto deste até o banheiro mais próximo (considerado como o mais utilizado) foram classificados como tendo "ótimas condições de acesso" pela maioria dos entrevistados (69\% e $81 \%$, respectivamente). O segundo andar em geral teve a mesma classificação ("ótimas condições de acesso") de acordo com $85 \%$ dos usuários. Em relação ao uso das dependências (tabela 3), o banheiro e os bebedouros obtiveram a classificação "ótimo, administro tudo sozinho" ( $81 \%$ e $92 \%$, respectivamente). Cabe destacar que nenhum dos 21 bebedouros presentes avaliados nas dependências da EEFFTO atende aos requisitos para acessibilidade de pessoas com MR (SOKI; COSTA, 2006). Além disso, $60 \%$ dos itens avaliados nos banheiros da EEFFTO foram considerados inacessíveis na pesquisa anterior (SOKI; COSTA, 2006). Tais resultados sugerem que as barreiras arquitetônicas não interferem na usabilidade dos indivíduos com MR nesses espaços. 
SALLES, B. G. et al. A acessibilidade arquitetônica. Rev. Ter. Ocup. Univ. São Paulo, v. 21, n. 1, p. 83-88, jan./abr. 2010.

Tabela 1 - Percepção dos usuários da acessibilidade do segundo andar da EEFFTO

\begin{tabular}{lcccc}
\hline \multicolumn{1}{c}{ Trajetos e espaços } & $\begin{array}{c}\text { Ótimas condi- } \\
\text { ções (\%) }\end{array}$ & $\begin{array}{c}\text { Boas condições, } \\
\text { mas necessita de } \\
\text { melhoras (\%) }\end{array}$ & $\begin{array}{c}\text { Condições de } \\
\text { acesso ruins. } \\
\text { Oferece muita } \\
\text { dificuldade (\%) }\end{array}$ & $\begin{array}{c}\text { Inacessível (\%) } \\
\text { Nunca utilizo } \\
(\%)\end{array}$ \\
\hline $\begin{array}{l}\text { Estacionamento - entra- } \\
\text { Entrada - CEPODE }\end{array}$ & 27 & 61,5 & 11,5 & - \\
CEPODE - banheiros & 69 & 27 & 4 & - \\
Banheiros & 81 & 11,5 & - & - \\
Segundo andar em geral & 58 & 27 & 7,5 & - \\
\hline
\end{tabular}

O uso da biblioteca (localizada no terceiro andar), dos elevadores e da cantina (localizada no primeiro andar) obtiveram como resposta predominante a opção "outro" $(92,5 \%, 77 \%$ e $50 \%$, respectivamente), o que, pelos relatos dos entrevistados, referia-se à não utilização do espaço. Este não uso/desconhecimento dos espaços também refletiu nas respostas referentes à acessibilidade, uma vez que não há como avaliar um ambiente sem o conhecer e utilizá-lo. Os trajetos do estacionamento do primeiro andar até a entrada do mesmo andar, do CEPODE até a cantina e do CEPODE até a biblioteca, além da acessibilidade dentro da cantina e da biblioteca, receberam a classificação "Nunca utilizo" pela maior parte dos entrevistados $(61,5 \%, 50 \%, 84,5 \%, 54 \%$ e $92 \%$, respectivamente). A classificação da acessibilidade do primeiro e terceiro andar em geral também foi "Nunca utilizo" para a maior parte dos indivíduos (46\% e $85 \%$, respectivamente). Porém, na classificação da acessibilidade da Escola em geral, $73 \%$ dos indivíduos classificaram como "Ótimas condições de acesso".

Tabela 2 - Percepção dos usuários da acessibilidade do primeiro (1A) e terceiro (3A) andares da EEFFTO

\begin{tabular}{|c|c|c|c|c|c|}
\hline Trajetos e espaços & $\begin{array}{l}\text { Ótimas condi- } \\
\text { ções }(\%)\end{array}$ & $\begin{array}{l}\text { Boas condições, } \\
\text { mas necessita de } \\
\text { melhoras }(\%)\end{array}$ & $\begin{array}{c}\text { Condições de aces- } \\
\text { so ruins. Oferece } \\
\text { muita dificuldade } \\
(\%) \\
\end{array}$ & Inacessível (\%) & Nunca utilizo (\%) \\
\hline Estacionamento - entrada do $1^{\circ}$ andar & 27 & 7,5 & 4 & - & 61,5 \\
\hline CEPODE - cantina ( $\left(1^{\mathrm{a}}\right)$ & 34,5 & 11,5 & 4 & - & 50 \\
\hline Cantina $\left(1^{\mathrm{a}}\right)$ & 27 & 15 & 4 & - & 54 \\
\hline CEPODE - biblioteca ( $3^{\mathrm{a}}$ ) & 4 & 7,5 & 4 & - & 84,5 \\
\hline Biblioteca ( $\left.3^{a}\right)$ & 4 & 4 & - & - & 92 \\
\hline Elevadores & 19 & 4 & - & - & 77 \\
\hline Primeiro andar em geral & 31 & 19 & 4 & - & 46 \\
\hline Terceiro andar em geral & 7,5 & 7,5 & - & - & 85 \\
\hline
\end{tabular}

Tabela 3 - Percepção dos usuários quanto ao seu desempenho nos espaços da EEFFTO

\begin{tabular}{|c|c|c|c|c|c|}
\hline Espaços & $\begin{array}{l}\text { Ótimo, administro } \\
\text { tudo sozinho }(\%)\end{array}$ & $\begin{array}{l}\text { Bom, administro qua- } \\
\text { se tudo sozinho }(\%)\end{array}$ & $\begin{array}{c}\text { Regular, administro } \\
\text { algumas coisas sozinho } \\
(\%)\end{array}$ & $\begin{array}{l}\text { Ruim, preciso de ajuda na } \\
\text { maioria das coisas }(\%)\end{array}$ & $\begin{array}{c}\text { Outro } \\
(\%)\end{array}$ \\
\hline Banheiros & 81 & 7,5 & - & 4 & 7,5 \\
\hline Telefones públicos & 46 & - & - & 4 & 50 \\
\hline Bebedouros & 92 & - & 4 & - & 4 \\
\hline Biblioteca & 7,5 & - & - & - & 92,5 \\
\hline Elevadores & 23 & - & - & - & 77 \\
\hline Cantina & 46 & 4 & - & - & 50 \\
\hline
\end{tabular}


Apesar de considerarem as condições de acesso como sendo ótimas em sua maioria, os usuários têm reivindicações. Dentre as sugestões feitas pelos entrevistados para melhoria da acessibilidade de indivíduos com MR ao prédio, incluemse: a pavimentação asfáltica do estacionamento do segundo andar; a adequação do piso do segundo andar do prédio com antiderrapantes; a diminuição da inclinação da rampa para a piscina; a instalação de corrimão nas escadas; a construção de banheiros adaptados mais próximos ao CEPODE; a construção de rampas no estacionamento; o deslocamento dos caixas bancários (que se encontram no terceiro andar) para o segundo andar; e a instalação de telefone público em altura acessível. Constata-se, desse modo, a relevância da usabilidade: apesar de os resultados apontarem para o não comprometimento da acessibilidade, os usuários tiveram a oportunidade de expressar algumas sugestões que podem favorecer o melhor uso do espaço, de forma a garantir a relação entre o componente pessoal (motivação, componente funcional, hábitos, papéis, interesses), o componente ambiental (arquitetônico) e o componente atividade. Apesar de os resultados não apontarem para o comprometimento da usabilidade pelas barreiras arquitetônicas, a possibilidade de melhorias ambientais pode vir a favorecer, ainda mais, a atividade e participação dos usuários com mobilidade reduzida na EEFFTO.

O resultado encontrado indica que a acessibilidade parece não interferir na usabilidade, entretanto, deve-se ressaltar que foi utilizada a amostra que freqüenta o CEPODE, ou seja, todos conseguiam de alguma forma se locomover e utilizar vários espaços da Escola, mesmo que com alguma dificuldade. Porém, faz-se necessário uma pesquisa com ex-usuários do CEPODE para averiguar os motivos que os levaram ao abandono do projeto, para se investigar se as condições de acessibilidade interferiram nessa decisão.

Outro aspecto que merece destaque é a questão da subjetividade das respostas dos entrevistados. Como explicado anteriormente, a usabilidade é interpretada mais subjetivamente, o próprio indivíduo julgará o grau de comprometimento que o ambiente lhe traz na realização de suas atividades (FANGE; IWARSSON, 2003). Dessa forma, outros fatores, além do ambiente físico da Escola propriamente dito, podem ter influenciado as respostas. Uma possibilidade é o fato de os ambientes que o indivíduo costuma freqüentar (sua casa e proximidades, por exemplo) poderem apresentar situações mais precárias de acessibilidade do que a Escola e, dessa forma, esta última ser julgada como tendo ótima acessibilidade. Muitos parecem gostar muito do trabalho realizado no CEPODE, por esse motivo alguns podem também tender a julgar a acessibilidade da Escola de forma mais favorável do que é realmente. Devido a isso, sugere-se dar continuidade a esse estudo, utilizando a metodologia qualitativa, com o intuito de descobrir o que levou esses indivíduos a responderem que o prédio está em ótimas condições de acesso, sendo que muitas barreiras arquitetônicas foram confirmadas (SOKI; COSTA, 2006). Além disso, estudos sobre a acessibilidade desses indivíduos em seu ambiente doméstico seriam convenientes, no sentido de investigar se a percepção positiva que eles têm das condições de acessibilidade da EEFFTO têm relação com a possível dificuldade de acesso que esses porventura constatem em suas próprias casas.

Considerando todas as sugestões feitas nas entrevistas, objetiva-se consolidar ações que garantam a acessibilidade plena de pessoas com deficiência na Escola. Para isso, pretende-se sensibilizar a direção da Escola para enviar uma proposta para o Programa Incluir, do Ministério da Educação, que "tem por objetivo promover ações que garantam o acesso e permanência de pessoas com deficiência nas Instituições Federais de Educação Superior; apoiar a criação, reestruturação e/ou consolidação de núcleos de acessibilidade nas instituições federais de ensino superior; implementar a política de acessibilidade plena de pessoas com deficiência na educação superior; promover a eliminação de barreiras pedagógicas, atitudinais, arquitetônicas e de comunicações e a efetivação da política de acessibilidade universal” (MEC, 2009).

\section{CONCLUSÃO}

A aplicação de questionário que contemplasse a usabilidade garantiu o levantamento do entendimento do ambiente pelos próprios usuários, considerando-se os componentes pessoais, ambientais e da atividade. Diante dos resultados e discussão deste artigo, conclui-se que a acessibilidade parece não interferir na usabilidade de indivíduos com mobilidade reduzida na EEFFTO. Entretanto, a melhoria das condições de acesso auxilia na inclusão desses usuários no espaço da Escola.

Uma vez que a EEFFTO abriga cursos da área de reabilitação, que formam profissionais que atuam diretamente na promoção da inclusão de pessoas com deficiência, ela poderia ser uma referência para as demais Escolas da UFMG. Desse modo, constatadas as irregularidades arquitetônicas e expressas as sugestões de usuários atendidos no CEPODE, essas sugestões podem ser discutidas e implementadas, de modo a proporcionar melhores condições de acesso e uso do espaço da Escola.

Sugere-se que seja realizada uma pesquisa que vislumbre os ex-usuários que abandonaram o serviço nos últimos dois ou três anos, para avaliar se a acessibilidade contribuiu ou não para tal decisão. 
SALLES, B. G. et al. A acessibilidade arquitetônica. Rev. Ter. Ocup. Univ. São Paulo, v. 21, n. 1, p. 83-88, jan./abr. 2010.

Agradecimento: O apoio da coordenação do CEPODE e as contribuições de seus usuários, que tornaram essa pesquisa possível.

SALlES, B. G.; GUERRA, F. P.; SOKI, E. A.; COSTA, M. L. G.; REZENDE, M. B. Does accessibility interfere in usability of people with restricted mobility? Rev. Ter. Ocup. Univ. São Paulo, v. 21, n. 1, p. 83-88, jan./abr. 2010.

\begin{abstract}
One of the goals of the Occupational Therapy's intervention is to guarantee the inclusion of the individuals in all aspects of their lives with the maximum of independence and autonomy. Beyond the avaliation of the physical environment (accessibility), the professional must verify the usability, which is the persons' subjective avaliation of the degree in which the environment restricts their performance. This study was made with the objective to investigate, under the individuals with restricted mobility's point of view, if the architecture conditions interfere in their activities and participation in the Escola de Educação Física, Fisioterapia e Terapia Ocupacional (EEFFTO) of the Universidade Federal de Minas Gerais (UFMG), to verify their satisfaction about UFMG's support and their suggestions of adaptation. For that, a mixed and descriptive study (survey type) was developed. A questionnaire was applied between May and September of 2008 with 26 persons with restricted mobility. The questionnaire had 4 descriptive questions and 21 multiple choice questions. The initial questions aimed to describe the sample, the others broached the access and use of the physical environment. In the accessibility's classification, $73 \%$ of the individuals considered that the EEFFTO in general had "Great conditions of access". This shows their satisfaction with the access conditions of the building, although some irregularities had already been evidenced; so the architecture barriers don't interfere in the usability of these individuals.
\end{abstract}

KEY WORDS: Mobility limitations. Architectural accessibility/standards. Handicapped advocacy. Disability evaluation. Health services accessibility.

\title{
REFERÊNCIAS
}

1. ASSOCIAÇÃO BRASILEIRA DE NORMAS TÉCNICAS. NBR 9050: acessibilidade a edificações, mobiliário, espaços e equipamentos urbanos. Rio de Janeiro, 2004.

2. BRITO, L.S., et al. Acessibilidade de cadeirantes em clínicas de fisioterapia do plano piloto de Brasília-DF. Universitas: Ciên. Saúde, v. 4, n.1/2, p. 17-35, 2006.

3. FANGE, A.; IWARSSON, S. Accessibility and usability in housing construct validity and implications for research and practice. Disabil. Rehabil., v. 25, n. 23, p. 1316-1325, 2003

4. IWARSSON, S.; STAHL, A. Accessibility, usability and universal design - positioning and definition of concepts describing person-environment relationships. Disabil. Rehabil., v. 25, n. 2, p. 57-66, 2003.
5. RIBEIRO, M. A. Design universal. In: CAVALCANTI, A.; GALVÃO, C. Terapia ocupacional fundamentação e prática. Rio de Janeiro: Guanabara Koogan, 2007. p. 417-19.

6. SOKI, E. K.; COSTA, M. L. G. Fatores ambientais que interferem na acessibilidade de usuários com mobilidade reduzida na Escola de Educação Física, Fisioterapia e Terapia Ocupacional da Universidade Federal de Minas Gerais. 2007. 96f. Monografia (conclusão do curso) - Universidade Federal de Minas Gerais, Escola de Educação Física, Fisioterapia e Terapia Ocupacional, Belo Horizonte.

7. Programa incluir: Disponível em: http://portal.mec.gov.br/sesu/ index.php?option $=$ content $\&$ task $=$ view $\& i d=557 \&$ Itemid $=303$. Acesso em 16 jan. 2009. 